\title{
HERMITE-HADAMARD-TYPE INEQUALITIES IN THE APPROXIMATE INTEGRATION
}

\author{
SZYMON WĄSOWICZ
}

Abstract. We give a slight extension of the Hermite-Hadamard inequality on simplices and we use it to establish error bounds of the operators connected with the approximate integration.

Mathematics subject classification (2000): 26D15, 41A80, 65D32, 15A63, 26B25, 39B62.

Key words and phrases: Approximate integration, Hermite-Hadamard inequality, convex functions, cubatures, norm of a quadratic form.

\section{REFERENCES}

[1] M. BessenYeI, The Hermite-Hadamard inequality on simplices, Amer. Math. Monthly 115 (2008), 339-345.

[2] R. T. Rockafellar, Convex Analysis, Princeton University Press, Princeton, New Jersey, 1970.

[3] R. A. SHARIPOV, Course of Linear Algebra and Multidimensional Geometry, Publ. of Bashkir State University, Ufa 1996, online: http://uk.arxiv.org/abs/math/0405323v1

[4] S. WĄSOWICZ, Inequalities between the quadrature operators and error bounds of quadrature rules, J. Ineq. Pure \& Appl. Math. (JIPAM) 8 (2007), Article 42, online: http: //jipam.vu.edu.au

[5] S. WĄSOWICZ, On error bounds for Gauss-Legendre and Lobatto quadrature rules, J. Ineq. Pure \& Appl. Math. (JIPAM) 7 (2006), Article 84, online: http://jipam.vu.edu.au

[6] S. WĄSOWICZ, On quadrature rules, inequalities and error bounds, J. Ineq. Pure \& Appl. Math. (JIPAM), 9 (2008), Article 36, online: http://jipam.vu.edu.au 